\title{
Un enfoque narrativo sobre las personas inmigrantes en Costa Rica: utilización de una técnica cualitativa de origen japonés
}

\author{
A narrative approach towards immigrants in Costa Rica: using a \\ qualitative Japanese technique \\ Chikae Kasuya \\ Departamento de Psicología, Facultad de humanidades, \\ Iwaki Meisei University, Japòn. \\ Dirección postal: 5-5-1 Chuodai-iino, Iwaki, 970-8551, Japan. \\ Ce:kasuya@abox8.so-net.ne.jp \\ kasuya@iwakimu.ac.jp
}

\begin{abstract}
Resumen. Este artículo tiene como objetivo ofrecer una técnica cualitativa de origen japonés llamada kamishibai, la cual es un tipo de libro ilustrado. Se realizó una investigación-acción en una comunidad marginal de Costa Rica, y se hizo un kamishibai cuya historia consistió en el relato de vida de una anciana nicaragüense. Los miembros de su familia compartieron el proceso de redacción. El análisis se realizó desde un enfoque narrativo y se generaron hipótesis sobre las ventajas de la técnica "kamishibai de la vida". Las hipótesis generadas fueron: (1) se ubicarían las experiencias de la vida en el eje cronológico, (2) se evaluaría el significado de las experiencias en la vida desde el punto de vista de la informante, (3) la técnica funcionaría como forma de comunicación, (4) se transmitirían contenidos a quienes no pueden leer, por ejemplo, los(as) infants.
\end{abstract}

Palabras clave: enfoque narrativo, relato de vida, inmigrante nicaragüense.

Abstract. The present article has the objective of offering a qualitative technique of Japanese origin named kamishibai, which is a kind of picture book. An action-research was done at a marginal community of Costa Rica, and a kamishibai was made from the life story of an old Nicaraguan. Her family members participated in the production process. An analysis was done by a narrative approach, and some hypothesis about advantage of the technique "kamishibai of life" were generated. The hypothesis were: (1) they places informant's experiences of life in chronological order, (2) they attempt to understand the meaning of experiences from the perspective of the individual involved, (3) they function as a way of communication, (4) they convey contents to whom don't have the ability to read and understand, e.g. infants.

Key Words: narrative, life story, Nicaraguan immigrant. 
Este artículo tiene como objetivo ofrecer una técnica cualitativa de origen japonés llamada kamishibai. La palabra kamishibai significa, en japonés, "teatro de papel", es una forma de narrar cuentos, y se podría definir como un estilo de libro ilustrado. Este artículo reporta la experiencia de crear un kamishibai cuya historia se basa en la vida de una anciana nicaragüense que vive en una comunidad marginal de Costa Rica. En la primera sección se describe el enfoque narrativo desde la perspectiva que tratan los relatos. En la segunda se presenta qué es un kamishibai. En la tercera se informa sobre el proceso para hacer el kamishibai de la vida. Por último, se discute acerca de la eficiencia de esta técnica.

\section{Enfoque narrativo como método de práctica y método de investigación}

En los últimos años ha habido un creciente interés en el enfoque narrativo dentro del campo de la psicología, el bienestar social, la medicina y la enfermería como un método de práctica; a la vez, en el campo de las ciencias sociales como método de investigación. Noguchi (2009) dice que el objeto del enfoque narrativo no es analizar relatos, sino demostrar cómo funcionan estos en algunos fenómenos. La palabra narrativa tiene dos significados: "narrar" (acto) y "lo que se ha narrado" (producto hablado). Bruner (1986) propone que el "modo de pensamiento narrativo" es la modalidad más antigua del pensamiento humano. Al contar historias a otras personas, nuestras experiencias adquieren sentido. Es decir, el significado de las experiencias surge de la narración.

La función de la narrativa posee tres clasificaciones (Elliott, 2005): cronológica (chronologica), significativa (meaningful) y social (social). En primer lugar, la clasificación cronológica quiere decir que la narrativa organiza experiencias en nuestra vida en el orden en que sucedieron. Por lo general, vivimos con varias experiencias desordenadas, pero una vez que intentamos contarlas a otras personas, mencionamos varios acontecimientos indicando cuándo ocurrieron, de esta manera los acontecimientos llegan a ser colocados en el eje cronológico.

En segunda instancia, la clasificación significativa se da cuando el narrador o narradora brinda el sentido a sus experiencias. Las experiencias en la vida son polisémicas y hay varias maneras de ver una experiencia. ¿Cómo afecta una experiencia la vida? Cuando hacemos un relato de nuestra vida, ubicamos las influencias de nuestros acontecimientos en esta y tratamos de contar la vida como una historia con consistencia, esto es importante para la identidad. Nuestra identidad es un compuesto social que no se considera como algo fijo, sino que está en constante creación y revisión. Erikson, Erikson \& Kivnic (1990) dicen que el proceso narrativo organiza los 
acontecimientos con la continuidad del propio ser, al tiempo que contribuye a mantener y renovar la identidad.

En tercer lugar, la clasificación social surge cuando la actividad de narrar funciona como una forma de interacción con otras personas. Bruce (1999) estudia el valor de reminiscencia en la vejez y dice que la adaptación psicológica social es promovida por contar relatos a otras personas e intentar conseguir su comprensión. En el proceso narrativo no puede faltar la presencia de otra persona que escucha, la cual tiene interés por la vida de quien narra. Se puede decir que la presencia de otra persona es lo más importante para el proceso narrativo. En cuanto a talleres de rehabilitación de memoria en ancianos, la técnica narrativa tiene un doble efecto benéfico: en sí mismo por el esfuerzo de refrescar la memoria y, por otra parte, pues facilita el fortalecimiento de redes sociales.

Compartir lo que se ha narrado con otras personas contribuye a dar la realidad social a la historia. Es decir, los "otros" pasan a ser "testigos" de la historia. Por ejemplo, en la Terapia Narrativa (White \& Epston, 1990) se entiende la terapia como un proceso conversacional entre clientes y terapeutas, en el que ambos dan nuevos significados a los acontecimientos de los(as) clientes y co-construyen soluciones al problema. Como parte del proceso de terapia, el o la terapeuta escribe cartas que mencionan lo que se ha contado entre ellos, o certificados que señalan lo que logra su cliente, y los obsequia a este (White \& Epston, 1990). El o la cliente comparte sus relatos con el o la terapeuta y siente concretamente la realidad que se construye por el proceso conversacional. Puede decirse que compartir relatos con terceras personas, que no son ni el narrador(a) ni el o la oyente, da una realidad social más concreta. Berger \& Luckmann (1966) señalan que la realidad del mundo cotidiano se construye socialmente. Una persona sabe todo lo que ocurre por la actitud que presentan otras personas ante determinados sucesos, y por otra parte, una persona se conoce a sí misma por la actitud que tienen los otros hacia ella. Se puede decir que para los(as) clientes es significativo compartir relatos con otras personas en la terapia. El o la terapeuta facilita la emergencia de relatos alternativos por varias maneras. Aunque no es equivalente a la terapia narrativa, la técnica de la historia en dibujos puede ser un recurso de este tipo de terapia, porque los dibujos facilitan compartir la historia de los(as) clientes así como estimular cognitiva y socialmente a las personas.

Es incierto si lo que narran expresa la verdad objetiva. Según Yamada (2000), el relato de vida (life story) se refiere al cuento sobre la vida realizado por la persona que la ha vivido, y describe la realidad subjetiva que se ha experimentado. Los cuentos de el o la informante se convertirán en el relato de vida. Se considera que es significativo estudiar cómo funciona el relato de vida para quien lo cuenta. 
En Japón, Okuda, trabajadora social de una institución para personas de la tercera edad, hace "kamishibai de la vida" como un regalo de cumpleaños para los ancianos y las ancianas y se los presenta a todos en la institución (Okuda, 2006). Aunque la práctica de Okuda se ubica en una parte de los servicios cotidianos para estas personas, parece que el proceso de hacer el kamishibai de la vida tiene funciones similares a la terapia narrativa, porque en esta los(as) terapeutas escuchan bien lo que narran los(as) clientes, y escriben cartas o certificados. Sin embargo, cabe señalar que el kamishibai de la vida consta no solo de escritos sino también de pinturas, mientras que por lo general el relato de vida está constituido únicamente por escritos. A pesar de que el kamishibai de la vida es una técnica original, no se ha estudiado su función. Por tanto, el objeto de este artículo es plantear algunas hipótesis sobre la eficiencia de la técnica desde el punto de vista del enfoque narrativo a través de una práctica en la cual se hace un kamishibai sobre la vida de una anciana nicaragüense en Costa Rica. El motivo por el cual se seleccionó a esta mujer es porque las personas inmigrantes, en teoría, tienen diversas dificultades en la vida aun cuando no sufran enfermedades mentales.

\section{¿Qué es kamishibai?}

Se puede considerar que contar historias mostrando dibujos es una de las formas más comunes en distintas culturas desde tiempos muy antiguos. En Japón, por ejemplo, se puede ver en una escena de Genji Monogatari Emaki que fue dibujado en el siglo XII. Sin embargo, el kamishibai en su forma actual fue construido en el año 1930 (Uechi, 1997). Este fue conocido como "kamishibai en la calle" (gaito-kamishibai), el cual se presentaba a los(as) niños(as) en las calles o parques; los(as) presentadores les vendían dulces a los(as) infantes, es decir, presentaban los kamishibai para vendérselos. El Kamishibai en la calle tenía dibujos sensacionales a fin de que llamaran la atención de muchos niños y niñas, y también tenían una gran variedad de historias: historias de aventura, historias de fantasmas, historias dramáticas, historias cómicas, entre otras. En los años 30, cuando el kamishibai en la calle se hacía cada vez más popular, los predicadores religiosos y pedagogos(as) de enseñanza infantil empezaron a prestar atención al gran efecto del kamishibai como medio para comunicar y empezaron a hacer sus propios kamishibai con historias educativas (Ishiyama, 2008). Primero hicieron un kamishibai de la historia de la Biblia para predicar la religión cristiana y después kamishibais para la educación infantil y la educación social. Estos kamishibais se llamaban "kamishibai educativo" (kyöiku-kamishibai). Durante la Segunda Guerra Mundial, el gobierno de Japón hizo kamishibais a fin de elevar la moral de lucha, o sea "kamishibai para la política nacional" (kokusaku-kamishibar) (Suzuki, 2005). Después de la guerra y, hasta cierto punto, arrepentidos por 
el "kamishibai para la política nacional", hicieron kamishibai para enseñar la democracia y el pacifismo. De este modo, los diversos kamishibais, independientemente de lo que se desea transmitir, indican la valoración de esta técnica como un medio educativo (Ishiyama, 2008). El inicio de la televisión en el país, en la década de los 50 fue una de las causas de decadencia inmediata del kamishibai; sin embargo, el "kamishibai educativo" sigue funcionando en el campo de la educación infantil, mientras que el "kamishibai en la calle", que fascinaba a niños y niñas, ha revivido recientemente por acontecimientos especiales, ya que tiene un contenido que se asemeja al "cómic japonés" (manga) y a la "animación japonesa" (anime) ${ }^{1}$; el "kamishibai educativo", en cambio, tiene un contenido similar a los libros ilustrados para niños y niñas. Hoy en día, en general, la palabra kamishibai se utiliza como sinónimo de "kamishibai educativo".

En los últimos años, el kamishibai se ha desarrollado y presentado en otros países. The International Kamishibai Association of Japan (IKAJA), que se organizó en 2001, promueve su divulgación en otras naciones. Por ejemplo, en España, Francia y Alemania se dan clases de kamisbibai (Nosaka, 2007), al tiempo que en países como Vietnam y Laos, se han formado autores de kamishibai, y se publican las obras de personas de la localidad (Matsui, 1998).

Una obra de kamisbibai consiste en un conjunto de 8 o 16 láminas. Cada lámina normalmente tiene 27 centímetros de largo y 38 de ancho. En un lado de la lámina hay dibujos grandes y simples lo cual permite que se puedan ver desde cierta distancia, ya que el kamishibai se presenta ante una audiencia. Por el otro lado de la lámina hay frases. Las historias de los kamishibai son simples, con frases cortas e incluyen diálogos. El presentador o presentadora no se limita a la lectura del texto, sino que lo interpreta para presentarlo como una obra. Además, cambia el estilo de presentación ajustándose a las reacciones de las distintas audiencias. Uno de los aspectos más importantes del kamishibai es la interacción entre la persona que lo presenta y el público. Algunos kamishibais tienen preguntas a la audiencia, a las cuáles deben contestar.

Para la audiencia, la forma del kamishibai se asemeja a la animación pues se observan las láminas, una tras otra, mientras se escucha la narración de la historia ${ }^{2}$. Unas presentadoras de kamishibai señalan que este crea "simpatía" y "concentración" en la audiencia (Nosaka, 2007; Matsui, 1998). Según Matsui (1998), cuando la audiencia comparte la misma historia se crean condiciones que permiten introducirse en el mundo en el cual esta fue creada, gracias a la

1 El "Kamishibai en la calle" llegó a su edad de oro antes del cómic y la animación japoneses. Se dice que este tipo de kamishibai ha tenido una gran influencia en ambas técnicas actuales. Algunos pintores del "kamishibai en la calle", que tuvo su ocaso en los 50, encontraron trabajo en el área del cómic japonés.

2 En los inicios de la televisión, la llamaban "kamishibai eléctrico". 
aparición de una imagen tras otra. Con respecto a la concentración, Kodaira (2007) captó las reacciones de niños y niñas $(\mathrm{N}=27$, años 4-5) cuando estaban mirando un kamishibai en el kínder. Cuando la historia llegó a su clímax hubo menos reacciones físicas, y en apariencia los niños y las niñas se habían metido en la historia como si no hubiera nadie más alrededor. Faltan estudios positivos como el de Kodaira (2007), que estudien la manera en que funciona el kamishibai.

El kamishibai destaca por su sencillez, no se necesita ninguna máquina y se puede utilizar en cualquier sitio. Nonaka et al. (2008) utilizaron kamishibai para la educación preventiva contra la malaria en Laos. Los (as) participantes fueron 130 alumnos(as) entre $3^{\text {er }}$ y $5^{\text {to }}$ grado de dos escuelas. El héroe de la historia es un estudiante de una escuela que aprende, a través de accidentes cotidianos (la muerte de su amigo, el sufrimiento de su papá, etc.), información acerca de la malaria, las técnicas para prevenirla y los comportamientos para propiciar la salud. Los investigadores entregaron kamishibai en blanco y negro a los estudiantes y ellos los colorearon. Después pidieron que los niños y niñas presentaran el kamishibai a su familia o a sus vecinos. Este estudio mostró que los estudiantes de las escuelas tenían el potencial de llevar los mensajes sobre la salud a sus comunidades. Una ONG japonesa Japanese Organization for International Cooperation in Family Planning (JOICFP) ha hecho kamishibai en Tanzania para prevenir los daños del SIDA y minimizar los prejuicios y discriminaciones contra portadores del VIH. Dicen que la presentación del kamishibai acelera el intercambio de opiniones acerca del VIH entre las habitantes de los pueblos, es decir, el kamishibai prepara la oportunidad para que lo comprendan desde varios puntos de vista.

Por otra parte, el kamishibai es sencillo porque la mayoría de sus dibujos son simples, no entran en detalles, y no es necesario encuadernarlo porque un conjunto de kamishibai está formado por láminas grandes. Estos aspectos facilitan la creación de kamishibais. Aunque la mayoría de kamishibais son hechos por casas editoriales, también hay kamishibais caseros. ¿Por qué se hace un kamishibai casero? Según Tokiwa (2007), se hacen para compartir algo con otras personas, por ejemplo, sentimientos (diversión, alegría), valores morales (el valor de la vida, la importancia de la amabilidad) y leyendas del lugar. La ventaja del kamishibai casero es que se puede adaptar según la necesidad. La principal característica del kamishibai es, como ya se ha mencionado, la sencillez, y el hecho de que permite diversas utilizaciones en cualquier lugar. 


\section{Hacer el kamishibai de la vida de una inmigrante nicaragüense: Método y resultado}

Se realizaron entrevistas no-estructuradas en una comunidad marginal ubicada en el área metropolitana de San José, Costa Rica en el año 2009. En este texto, como estudio de caso, se da el reporte de una informante, una septuagenaria anciana nicaragüense. Ella había vivido en Costa Rica por más de diez años y habitaba la comunidad en compañía de una hija y sus nietos. Se realizaron tres entrevistas en su casa, por alrededor de hora y media en total. La informante contó sobre la vida en Nicaragua y en Costa Rica. Su historia se registró en el cuaderno. Los miembros de su familia (hija y dos nietos) también participaron en el proceso, escuchando los cuentos de la informante y añadiendo detalles a la historia. La informante contó lo que quería y en el orden que quería. Cuando no podía seguir su narración, la investigadora le ayudó con preguntas como: “¿cómo eran esos días?", "¿cómo se sentía usted cuando...?”, ¿qué hizo usted después de...?, etc. Antes de la primera entrevista, se le indicó a la informante que no sería necesario contar todas las experiencias de su vida.

Los cuentos se hicieron en un kamishibai. El proceso de conformación del kamishibai fue el siguiente:

1. Planear la historia y los dibujos.

Puede usarse un modelo repetitivo, el cual crea el ritmo para la historia y hace que esta sea más clara. Es recomendable que la composición del cuadro sea sencilla, no es necesario dibujar detalles. Generalmente las láminas de papel se van pasando desde el lado derecho del presentador o la presentadora. Si hay algo que se quiere mostrar más adelante en una página, puede dibujarse por el lado derecho del papel.

2. Dibujar en papeles con lápiz.

3. Dibujar con marcador y pintar los dibujos con crayón. Se puede dibujar y pintar con cualquier material para colorear.

4. Escribir la historia en la otra cara. La historia se escribe en el revés de la página anterior. Cuando se presenta, las láminas del kamishibai se ponen una encima de la otra. Al principio, se muestra la lámina que tiene el dibujo de la primera página a la audiencia, y el presentador o presentadora lee la historia que está en el revés de la última página. Después, se retira la primera página y se coloca al final. La audiencia ve el dibujo de la segunda página y el presentador o presentadora lee la historia que está por detrás de la primera página.

Luego de hacer el kamishibai, la informante vio la presentación de este junto a su familia. Sus familiares hicieron comentarios entre ellos mientras se 
les presentaba. El kamishibai se le regaló a la informante. Según lo observado, los familiares quedaron muy satisfechos con el proceso y consideraron el kamishibai como un importante regalo para la anciana ${ }^{3}$. En el proceso de la técnica, la informante se comunicó con su familia y la investigadora quienes mostraron interés en su vida. Aunque es cierto que la protagonista del proceso fue la informante, puede decirse que la colaboración entre la participante, su familia y la investigadora concretó el relato de vida. Es posible que la experiencia de la colaboración haya aumentado la calidad de vida (quality of life) de la informante, pues la familia y la investigadora compartieron la vida de la informante y pusieron atención a esta.

El siguiente kamishibai fue hecho modificando detalles para salvaguardar la información personal de la informante (Figura 1).

Figura 1. El kamishibai de la vida

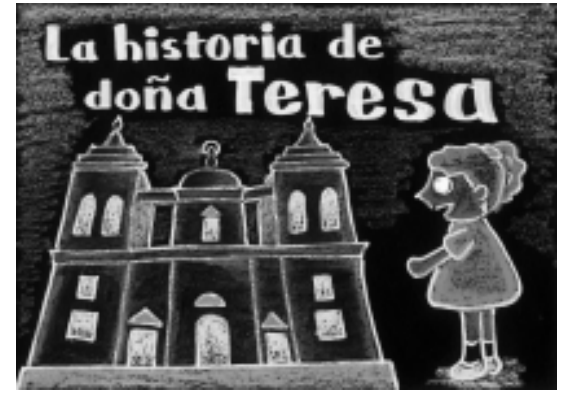

(1) Hace muchos años, en León, Nicaragua, vivía una niña que se llamaba Teresa. En León hay iglesias de hermosa arquitectura. ¿Conoce usted León? ¿Dónde está León?

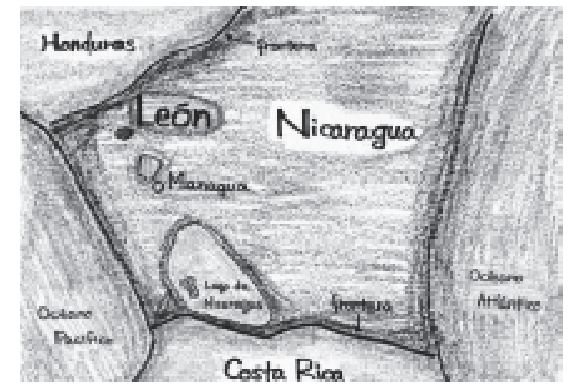

(2) Nicaragua está al norte de Costa Rica. Aquí está León. León fue fundada en 1524 y fue una de las primeras ciudades europeas en territorio continental americano. León es un importante centro industrial $y$ de comercio de Nicaragua.

\footnotetext{
${ }^{3} \mathrm{El}$ ambiente no facilitó la medición de los efectos que tuvo en la anciana y en su familia el haber revisado y compartido su historia. Por tanto, se reporta solo lo considerado producto de la observación.
} 

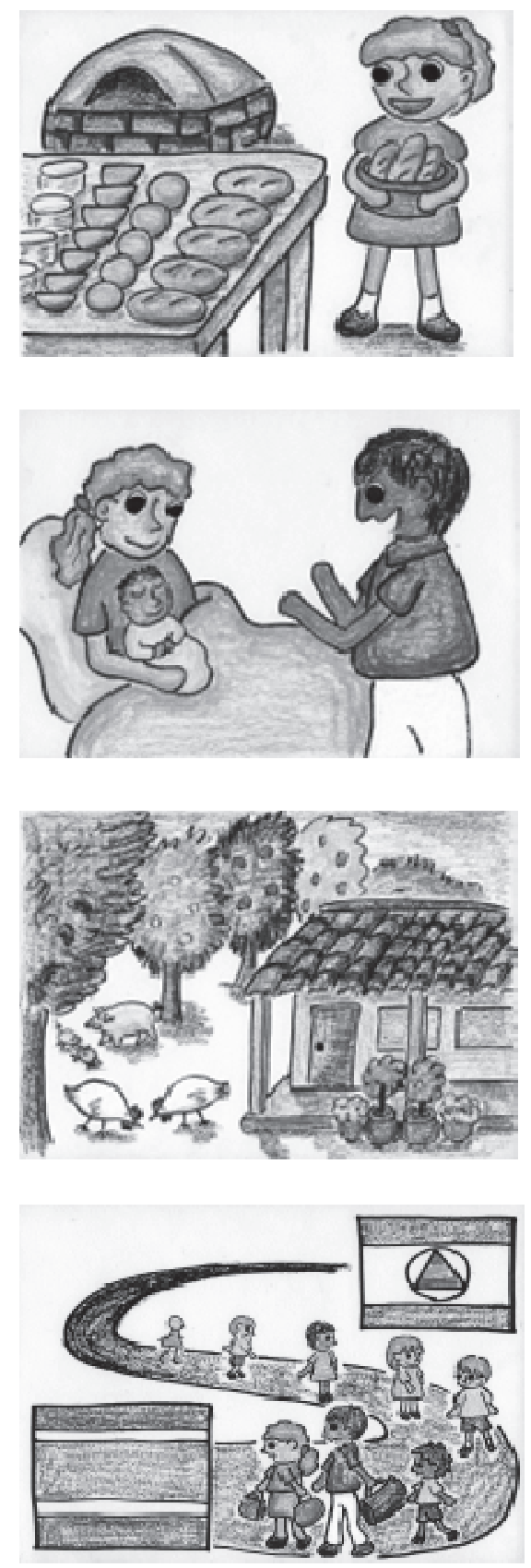

(3) La niña Teresa vivía con una señora. Teresa hacía muchas variedades de pan: pan simple, pupusas, empanadas y tortillas, $y$ los vendía.

(4) Cuando Teresa tenía 20 años, nació su primer hijo Miguel. El papá del bebé se llamaba Julio. Después, siguieron naciendo 5 hijos más: Fernando, Marta, Paula, Joaquín, y Sara.

(5) La familia vivía en una casita. En el jardín había flores y árboles de mango, naranja, y limón. Doña Teresa lavaba ropa, era lavandera. $\mathrm{Su}$ esposo era agricultor. Ellos trabajaban mucho todos los días.

(6) En 1994, la familia llegó a Costa Rica. Primero, doña Teresa sola, después toda la familia. 

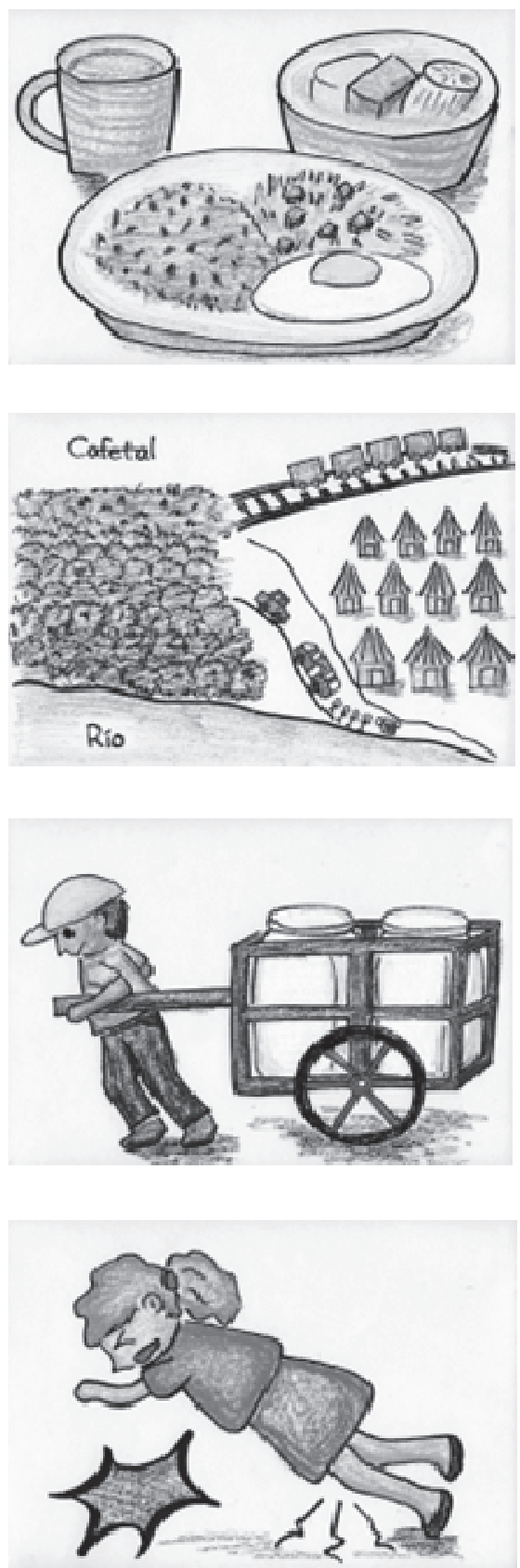

(7) Teresa empezó a trabajar en una soda. En la soda se servía olla de carne, sopa, gallo pinto, agua dulce, etc.

(8) En este año, 1994, la familia llegó a "La Candelaria". En aquella época "La Candelaria" era puro cafetal. El techo de la casa era de paja, y la pared era de bambú. No había ni luz ni agua.

(9) Iban al canal 6 para llevar agua tres veces por día, en una pichinga grande que pesaba muchísimo, la llevaba con una carreta. Para lavar la ropa, tenían que caminar dos kilómetros hasta donde había un chorro de agua. Muchas personas llegaban allá a hacer fila.

(10) En 2007, doña Teresa se cayó, y los doctores le hicieron una operación. 


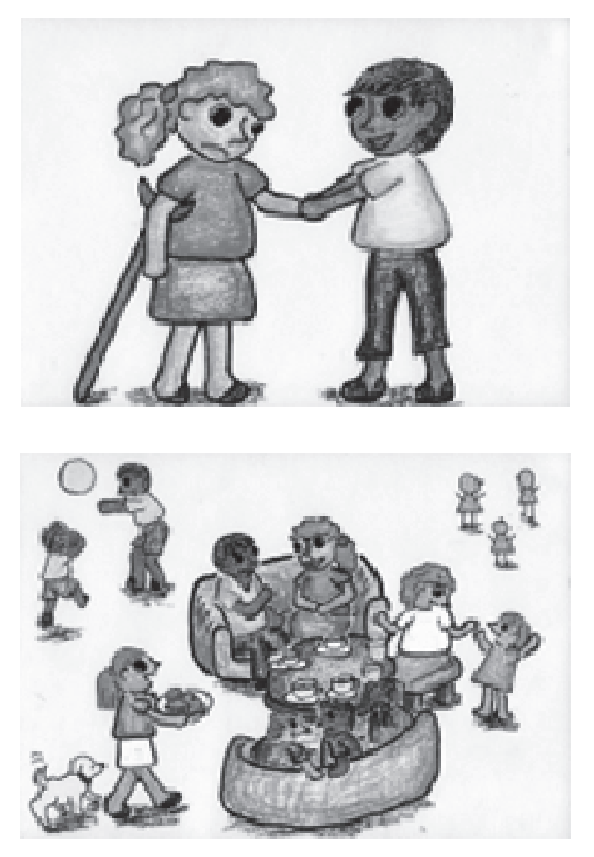

(11) El accidente la dañó, pero gracias a la gran ayuda de sus hijos doña Teresa se recuperó.

(12) Ahora doña Teresa tiene 28 nietos, y vive feliz al lado de su familia.

\section{Discusión}

Este estudio ha presentado el proceso de la técnica "kamishibai de la vida". Debido a que el estudio consiste en un solo caso, no es suficiente para juzgar las eficiencias de la técnica. Sin embargo, si se realizan nuevos estudios del mismo tema con más casos, se aclararía el funcionamiento de la técnica. Para saber cómo funciona el proceso de hacer un kamishibai de la vida, es necesario hacer más kamishibais y captar los sentimientos de los informantes en el proceso.

Aquí se indican las ventajas de la técnica como hipótesis para siguientes estudios. A causa de que el kamishibai consiste en texto y pintura, las ventajas son clasificadas en dos: las ventajas por texto y las ventajas por pintura.

En primer término, las ventajas por texto corresponden a tres funciones de la narrativa según Elliott (2005): cronológica, significativa y social.

(1) Se ubicarían las experiencias de la vida en el eje cronológico (Cronológica).

La anciana había experimentado varios sucesos y es posible que las experiencias se encontraran desordenadas en su memoria. Al contarlas, 
se escogieron las experiencias más importantes para la informante y se colocaron de manera ordenada.

(2) Se evaluaría el significado de las experiencias en la vida desde el punto de vista de la informante (Significativa).

Aunque los sucesos en el kamishibai eran solo una parte de la vida, lo importante es que la mayoría de las experiencias en el relato eran deseables para la informante o los sucesos que habían sido superados. Se considera que la realidad social del relato de vida se hizo más concreta, pues la compartieron su familia y la investigadora. Es decir, se considera que el relato obtuvo la realidad deseable.

(3) La técnica funcionaría como forma de comunicación (Social). En el proceso de la técnica, la informante se comunicó con su familia y la investigadora quienes mostraron interés en su vida. Aunque es cierto que la protagonista del proceso fue la informante, puede decirse que la colaboración entre la participante, su familia y la investigadora concretó el relato de vida. Es posible que la experiencia de la colaboración haya aumentado la calidad de vida (quality of life) de la informante, pues la familia y la investigadora compartieron la vida de la informante y pusieron atención a esta.

En segundo término, la ventaja por pintura.

(4) Se transmitirían contenidos a quienes no pueden leer, por ejemplo, los infantes.

La técnica del kamishibai pudo transmitir contenidos a los nietos de la informante. Se puede decir que esta tiene una capacidad de transmisión más amplia que otras técnicas que presentan solamente texto, aun el relato del kamishibai es muy sencillo.

Se puede añadir como ventaja secundaria, que el kamishibai de la vida se podría utilizar para hacer entender a los minorías marginadas, porque es una etnografía. La etnografía trata de inspirar la simpatía por otras personas o minorías marginadas para que se perciban como cercanas o que generen planes alternativos para cambiar su situación actual. Esta utilización no es nueva. El relato de vida, frecuentemente, hace de las minorías o grupos marginados el objeto de investigación y se han publicado varios estudios al respecto conteniendo narrativas. Con tal de hacer más kamishibais de la vida, se les utiliza en varias ocasiones.

\section{Agradeceimientos}

La autora desea agradecer a la Sra. Gail Nystrom de la Fundación Humanitaria Costarricense, a la Sra. Tatiana Blanco Sánchez de la Foundation for International Medical Relief of Children y a la Srta. Milagro 
Un enfoque narrativo hacia las personas inmigrantes en Costa Rica

Castro Solano del equipo editorial de la Revista Actualidades en Psicología por su valiosa colaboración en la elaboración de este artículo.

\section{Referencias}

Berger, P. \& Luckmann, T. (1966). The social construction of reality: A treatise in the sociology of knowledge. NY: Doubleday.

Bruce, E. (1999). Holding on to the story: Older people, narrative, and dementia. In G. Roberts, \& J. Holmes (Eds.) Healing stories: Narrative in psychiatry and psychology. Oxford University Press.

Bruner, J. (1986). Actual minds, possible worlds. Harvard University Press.

Elliott, J. (2005). Using narrative in social research: Qualitative and quantitative approaches. London: Sage.

Epston, D., \& White, M. (1992). A proposal for a reauthoring therapy: Rose's revisioning of her life and a commentary. In S. McNamee, \& K. J. Gergen (Eds.) Therapy as social construction. London: Sage.

Erikson, J. M., Erikson, E. H., \& Kivnic, H. (1990). Vital involvement in old age. NY: Norton.

Ishiyama, Y. (2008). Kamishibai bunkashi: Shiryō de yomitoku kamishibai no rekishi [The cultural history of Kamishibai: Kamishibai in historical materials]. Tokyo: Hōbun shorin.

Kodaira, H. (2007). Yōji no kamishibai he no han'nou: Han'nou pattern no chūshutsu to seikisuru bunmyaku no kentō [The child's reaction to Kamishibai: Patterns of reactions and shift with story line]. Annual Report of Studies, 29, 31-38. Japan: Nagoya Ryujo (St. Mary's) College.

Matsui, N. (1998). Kamishibai: Kōkan no yorokobi [Kamishibai: Pleasure of sympathy]. Tokyo: Dōshinsha.

Noguchi, Y. (2009). Narrative approach no tenkai [Development of narrative approach]. In Y. Noguchi (Ed.) Narrative approach (pp.1-25). Tokyo: Keisōshobō.

Nonaka, D., Kobayashi, J., Jimba, M., Vilaysouk, B., Tsukamoto, K., Kano, S., et al. (2008). Malaria education from school to community in Oudomxay province, Lao PDR. Parasitology International, 57, 76-82.

Nosaka, E. (2007). Sekai de sarani chikarazuyoku habataku tame ni: IKAJA no 5-nenkan wo tūjite [For diffusion at the world: Activity report of IKAJA for 5 years]. Kodomo no bunka, 39(7), 140-144. Tokyo: Kodomo no bunka kenkyūjo.

Okuda, M. (2006). Takurōjo "Min'na no ie" (Nishiizu-machi) ni okeru sōsaku kamishibai no torikumi [The practice of handmade kamishibai at a carehouse "Min'na no ie" (Nishiizu town)]. In A. Tōyama (Ed.) Hajimeyō rōjin care ni kamishibai: Mirukoto, tsukurukoto, enjirukoto no tanoshimi (pp.140-161). Tokyo: Kirara shobō.

Suzuki, T. (2005). Media to shite no kamishibai [Kamishibai as media]. Tokyo: Kyūzansha.

Tokiwa, H. (2007). Tezukuri kamishibai no sekai [The world of handmade kamishibai]. Kodomo no bunka, 39(7), 87-91. Tokyo: Kodomo no bunka kenkyūjo.

Uechi, C. (1997). Kamishibai no rekishi [The history of kamishibai]. Tokyo: Kyūzansha.

Yamada, Y. (2000). Jinsei wo monogataru: Seisei no life story [Tell the life: Generating life story]. Kyoto, Japan: Minerva shobō.

White, M., \& Epston, D. (1990). Narrative means to therapeutic ends. NY: Norton.

Recibido: 23 de mayo de 2010

Aceptado: 20 de agosto de 2011 